\title{
IJCARS Special Issue of the Digital Multiscale Patient: Marie Curie European Research Project on Multiscale Human
}

\author{
Nadia Magnenat Thalmann ${ }^{1}$
}

Published online: 9 May 2016

(C) CARS 2016

A special session has been held during CARS 2015 on the Digital Multiscale Patient. The papers presented were the results of the interdisciplinary research done during a Marie Curie European Research Project Multiscale Human coordinated by Professor Nadia Magnenat Thalmann. From 13 presentations during the special session, 6 papers have been selected for publication.

The first paper by Andra Chincisan and others, from MIRALab, University of Geneva, Switzerland, and LBBMHH, Hanover, Germany, presents a computational approach to calculate a personalized pennation angle based on MRI. This research is important as it allows to analyze personalized gait.

Matthias Becker et al., from MIRALab, University of Geneva in Switzerland, describe a model of multiresolution deformable meshes. The paper demonstrates the multimodal aspect of the segmentation.

The third paper deals with the semantic annotation of 3D anatomical models to support diagnosis and follow-up analysis of musculoskeletal pathologies. It is co-authored by
I. Banejee, and other researchers, all from CNR-IMATI, in Italy.

Another paper presented by Pitikakis and other authors, from Softeco, Italy, and MIRALab, University of Geneva, deals with automatic measurement and visualization of focal femoral cartilage thickness using 3D knee models.

Trombella et al., all from the hospital of Geneva in Switzerland, show the work done on 11C-acetate and PET/CT assessment of muscle activation in rat studies. The importance of this research in the multiscale aspect is to know the activity of muscle on rats before moving to human study.

The last paper co-authored by Assassi Lazhari and Nadia Magnenat Thalmann, both from MIRALab, University of Geneva, describes the assessment of cartilage contact pressure and loading in the hip joint during split posture.

We would like to thank Professor Heinz U. Lemke for his strong support in the organization of this special session of CARS 2015.
Nadia Magnenat Thalmann

thalmann@miralab.ch

1 MIRALab/C.U.I., University of Geneva, Battelle, 7, Route de Drize, 1227 Carouge, Geneva, Switzerland 